\title{
Some notes on commutators of the
} fractional maximal function on variable Lebesgue spaces

\author{
Pu Zhang ${ }^{1 *}\left({ }^{0}\right.$, Zengyan $\mathrm{Si}^{2}$ and Jianglong $\mathrm{Wu}^{1}$
}

"Correspondence:

puzhang@sohu.com

'Department of Mathematics,

Mudanjiang Normal University,

Mudanjiang, P.R. China

Full list of author information is

available at the end of the article

\begin{abstract}
Let $0<\alpha<n$ and $M_{\alpha}$ be the fractional maximal function. The nonlinear commutator of $M_{\alpha}$ and a locally integrable function $b$ is given by $\left[b, M_{\alpha}\right](f)=b M_{\alpha}(f)-M_{\alpha}(b f)$. In this paper, we mainly give some necessary and sufficient conditions for the boundedness of $\left[b, M_{\alpha}\right]$ on variable Lebesgue spaces when $b$ belongs to Lipschitz or $B M O\left(\mathbb{R}^{n}\right)$ spaces, by which some new characterizations for certain subclasses of Lipschitz and $B M O\left(\mathbb{R}^{n}\right)$ spaces are obtained.
\end{abstract}

MSC: 42B25; 42B20; 42B35; 46E30

Keywords: Fractional maximal function; Nonlinear commutator; Variable Lebesgue space; Lipschitz space; BMO space

\section{Introduction and main results}

Let $T$ be the classical singular integral operator. In 1976, Coifman, Rochberg and Weiss [4] studied the commutator generated by $T$ and a function $b \in B M O\left(\mathbb{R}^{n}\right)$ as follows:

$$
[b, T](f)(x)=T((b(x)-b(\cdot)) f(\cdot))(x)=b(x) T(f)(x)-T(b f)(x) .
$$

A well-known result states that $[b, T]$ is bounded on $L^{p}\left(\mathbb{R}^{n}\right)$ for $1<p<\infty$ if and only if $b \in B M O\left(\mathbb{R}^{n}\right)$. The sufficiency was obtained by Coifman, Rochberg and Weiss [4] and the necessity was proved by Janson [19]. Moreover, Janson also gave some characterizations of the Lipschitz space $\dot{\Lambda}_{\beta}\left(\mathbb{R}^{n}\right)$ (see Definition 1.2 below) via commutator $[b, T]$ in [19] and proved that $[b, T]$ is bounded from $L^{p}\left(\mathbb{R}^{n}\right)$ to $L^{q}\left(\mathbb{R}^{n}\right)$, for $1<p<n / \beta, 1 / p-1 / q=\beta / n$ and $0<\beta<1$, if and only if $b \in \dot{\Lambda}_{\beta}\left(\mathbb{R}^{n}\right)$ (see also Paluszyński [24]).

As usual, a cube $Q \subset \mathbb{R}^{n}$ always has its sides parallel to the coordinate axes. Denote by $|Q|$ the Lebesgue measure and $\chi_{Q}$ the characteristic function of $Q$. For $f \in L_{\text {loc }}^{1}\left(\mathbb{R}^{n}\right)$, we write

$$
f_{Q}=\frac{1}{|Q|} \int_{Q} f(x) d x
$$

(c) The Author(s) 2019. This article is distributed under the terms of the Creative Commons Attribution 4.0 International License (http://creativecommons.org/licenses/by/4.0/), which permits unrestricted use, distribution, and reproduction in any medium, provided you give appropriate credit to the original author(s) and the source, provide a link to the Creative Commons license, and indicate if changes were made. 
For $0 \leq \alpha<n$ and $f \in L_{\text {loc }}^{1}\left(\mathbb{R}^{n}\right)$, the fractional maximal function $M_{\alpha}$ is given by

$$
M_{\alpha}(f)(x)=\sup _{Q \ni x} \frac{1}{|Q|^{1-\alpha / n}} \int_{Q}|f(y)| d y,
$$

where the supremum is taken over all cubes $Q \subset \mathbb{R}^{n}$ containing $x$. When $\alpha=0$, we simply write $M$ instead of $M_{0}$, which is exactly the Hardy-Littlewood maximal function.

Similar to (1.1), we can define two different kinds of commutator of the fractional maximal function as follows.

Definition 1.1 Let $0 \leq \alpha<n$ and $b$ be a locally integrable function. The maximal commutator of $M_{\alpha}$ and $b$ is given by

$$
M_{\alpha, b}(f)(x)=\sup _{Q \ni x} \frac{1}{|Q|^{1-\alpha / n}} \int_{Q}|b(x)-b(y)||f(y)| d y,
$$

where the supremum is taken over all cubes $Q \subset \mathbb{R}^{n}$ containing $x$.

The nonlinear commutator of $M_{\alpha}$ and $b$ is defined by

$$
\left[b, M_{\alpha}\right](f)(x)=b(x) M_{\alpha}(f)(x)-M_{\alpha}(b f)(x)
$$

When $\alpha=0$, we simply denote by $M_{b}:=M_{0, b}$ and $[b, M]:=\left[b, M_{0}\right]$.

We call $\left[b, M_{\alpha}\right]$ the nonlinear commutator because it is not even a sublinear operator, although the commutator $[b, T]$ is a linear one. We would like to remark that the nonlinear commutator $\left[b, M_{\alpha}\right]$ and the maximal commutator $M_{\alpha, b}$ essentially differ from each other. For example, $M_{\alpha, b}$ is positive and sublinear, but $\left[b, M_{\alpha}\right]$ is neither positive nor sublinear.

The mapping property of $\left[b, M_{\alpha}\right]$ has been extensively studied; see $[1,2,8,12,13,16,23$, 25-31], for instance. There are some applications of nonlinear commutators in analysis. For example, $[b, M]$ can be used in studying the products of functions in $H^{1}$ and $B M O$ (see [3], for instance).

In 1990, by using the real interpolation techniques, Milman and Schonbek [23] obtained a commutator result, by which they obtained the $L^{p}$-boundedness of $[b, M]$ and $\left[b, M_{\alpha}\right]$ $(0<\alpha<n)$ when $b \in B M O\left(\mathbb{R}^{n}\right)$ and $b \geq 0$. In 2000, Bastero, Milman and Ruiz [2] considered the necessary and sufficient conditions for the boundedness of $[b, M]$ in $L^{p}\left(\mathbb{R}^{n}\right)$ when $b$ belongs to $B M O\left(\mathbb{R}^{n}\right)$. In 2009, Zhang and Wu [28] extended their results to commutators of the fractional maximal function. The results in [2] and [28] were extended to variable Lebesgue spaces in [29] and [30].

Recently, Zhang [26] studied the commutator $[b, M]$ when $b$ belongs to Lipschitz spaces. Some necessary and sufficient conditions for the boundedness of $[b, M]$ on Lebesgue and Morrey spaces are given. Some of the results were extended to variable Lebesgue spaces in [27] and to the context of Orlicz spaces in [15, 16] and [31].

Motivated by the papers mentioned above, in this paper, we mainly study the mapping properties of $\left[b, M_{\alpha}\right]$ in variable Lebesgue spaces when $b$ belongs to Lipschitz or $B M O\left(\mathbb{R}^{n}\right)$ spaces. More precisely, we will give some new necessary and sufficient conditions for the boundedness of $\left[b, M_{\alpha}\right]$ on variable Lebesgue spaces, by which some new characterizations for certain subclasses of Lipschitz and $B M O\left(\mathbb{R}^{n}\right)$ spaces are obtained. Moreover, our 
results also give affirmative answers to the questions mentioned in [16] and [29] (see Remarks 1.4 and 1.5 below, respectively). We would like to note that some of our results are new even in the case of Lebesgue spaces with constant exponents.

To state the results, we first recall some definitions and notations.

Let $\gamma \geq 0$, for a fixed cube $Q_{0}$, the fractional maximal function with respect to $Q_{0}$ of a locally integrable function $f$ is given by

$$
M_{\gamma, Q_{0}}(f)(x)=\sup _{\substack{Q \ni x \\ Q \subseteq Q_{0}}} \frac{1}{|Q|^{1-\gamma / n}} \int_{Q}|f(y)| d y,
$$

where the supremum is taken over all cubes $Q$ such that $x \in Q \subseteq Q_{0}$.

When $\gamma=0$, we simply write $M_{Q_{0}}$ instead of $M_{0, Q_{0}}$.

Definition 1.2 For $0<\beta<1$, we say a function $b$ belongs to the Lipschitz space $\dot{\Lambda}_{\beta}\left(\mathbb{R}^{n}\right)$, denoted by $b \in \dot{\Lambda}_{\beta}\left(\mathbb{R}^{n}\right)$, if there exists a constant $C>0$ such that for all $x, y \in \mathbb{R}^{n}$,

$$
|b(x)-b(y)| \leq C|x-y|^{\beta} .
$$

The smallest such constant $C$ is called the $\dot{\Lambda}_{\beta}$ norm of $b$ and is denoted by $\|b\|_{\dot{\Lambda}_{\beta}}$.

Definition 1.3 A locally integrable function $f$ is said to belong to $B M O\left(\mathbb{R}^{n}\right)$ if

$$
\|f\|_{B M O}:=\sup _{Q} \frac{1}{|Q|} \int_{Q}\left|f(x)-f_{Q}\right| d x<\infty
$$

where the supremum is taken over all cubes $Q$ in $\mathbb{R}^{n}$.

For a function $b$ defined on $\mathbb{R}^{n}$, we denote by

$$
b^{-}(x)= \begin{cases}0, & \text { if } b(x) \geq 0 \\ |b(x)|, & \text { if } b(x)<0\end{cases}
$$

and $b^{+}(x)=|b(x)|-b^{-}(x)$. Obviously, $b^{+}(x)-b^{-}(x)=b(x)$.

Definition 1.4 Let $p(\cdot): \mathbb{R}^{n} \rightarrow[1, \infty)$ be a measurable function. The variable Lebesgue space, $L^{p(\cdot)}\left(\mathbb{R}^{n}\right)$, is defined by

$$
L^{p(\cdot)}\left(\mathbb{R}^{n}\right)=\left\{f \text { measurable: } \int_{\mathbb{R}^{n}}\left(\frac{|f(x)|}{\lambda}\right)^{p(x)} d x<\infty \text { for some constant } \lambda>0\right\} .
$$

The set $L^{p(\cdot)}\left(\mathbb{R}^{n}\right)$ becomes a Banach space with respect to the norm

$$
\|f\|_{L^{p(\cdot)}\left(\mathbb{R}^{n}\right)}=\inf \left\{\lambda>0: \int_{\mathbb{R}^{n}}\left(\frac{|f(x)|}{\lambda}\right)^{p(x)} d x \leq 1\right\}
$$

We refer to $[5,10,21]$ and $[22]$ for more details on function spaces with variable exponents. 
Denote by $\mathscr{P}\left(\mathbb{R}^{n}\right)$ the set of all measurable functions $p(\cdot): \mathbb{R}^{n} \rightarrow[1, \infty)$ such that

$$
1<p_{-}:=\underset{x \in \mathbb{R}^{n}}{\operatorname{essinf}} p(x) \quad \text { and } \quad p_{+}:=\underset{x \in \mathbb{R}^{n}}{\operatorname{ess} \sup } p(x)<\infty
$$

and by $\mathscr{B}\left(\mathbb{R}^{n}\right)$ the set of all $p(\cdot) \in \mathscr{P}\left(\mathbb{R}^{n}\right)$ such that $M$ is bounded on $L^{p(\cdot)}\left(\mathbb{R}^{n}\right)$.

Remark 1.1 If $p(\cdot) \in \mathscr{B}\left(\mathbb{R}^{n}\right)$ and $\lambda>1$, then $\lambda p(\cdot) \in \mathscr{B}\left(\mathbb{R}^{n}\right)$. See Remark 2.13 in [6].

For notational convenience, we introduce a notation $\mathscr{B}^{\gamma}\left(\mathbb{R}^{n}\right)$ as follows.

Definition 1.5 Let $0<\gamma<n$. We say that an ordered pair of variable exponents $(p(\cdot)$, $q(\cdot)) \in \mathscr{B}^{\gamma}\left(\mathbb{R}^{n}\right)$, if $p(\cdot) \in \mathscr{P}\left(\mathbb{R}^{n}\right)$ with $p_{+}<n / \gamma$ and $1 / q(\cdot)=1 / p(\cdot)-\gamma / n$ with $q(\cdot)(n-\gamma) / n \in$ $\mathscr{B}\left(\mathbb{R}^{n}\right)$.

Remark 1.2 The condition $q(\cdot)(n-\gamma) / n \in \mathscr{B}\left(\mathbb{R}^{n}\right)$ is equivalent to saying that there exists a $q_{0}$ with $n /(n-\gamma)<q_{0}<\infty$ such that $q(\cdot) / q_{0} \in \mathscr{B}\left(\mathbb{R}^{n}\right)$. Moreover, $q(\cdot)(n-\gamma) / n \in \mathscr{B}\left(\mathbb{R}^{n}\right)$ implies $q(\cdot) \in \mathscr{B}\left(\mathbb{R}^{n}\right)$. See Remark 2.13 in [6] for details.

Our results can be stated as follows.

Theorem 1.1 Let $0<\beta<1,0<\alpha<n, 0<\alpha+\beta<n$ and let $b$ be a locally integrable function. Then the following statements are equivalent:

(1) $b \in \dot{\Lambda}_{\beta}\left(\mathbb{R}^{n}\right)$ and $b \geq 0$.

(2) $\left[b, M_{\alpha}\right]$ is bounded from $L^{p(\cdot)}\left(\mathbb{R}^{n}\right)$ to $L^{q(\cdot)}\left(\mathbb{R}^{n}\right)$ for some $(p(\cdot), q(\cdot)) \in \mathscr{B}^{\alpha+\beta}\left(\mathbb{R}^{n}\right)$.

(3) $\left[b, M_{\alpha}\right]$ is bounded from $L^{p(\cdot)}\left(\mathbb{R}^{n}\right)$ to $L^{q(\cdot)}\left(\mathbb{R}^{n}\right)$ for all $(p(\cdot), q(\cdot)) \in \mathscr{B}^{\alpha+\beta}\left(\mathbb{R}^{n}\right)$.

(4) There exists an $s(\cdot) \in \mathscr{B}\left(\mathbb{R}^{n}\right)$ such that

$$
\sup _{Q} \frac{1}{|Q|^{\beta / n}} \frac{\left\|\left(b-|Q|^{-\alpha / n} M_{\alpha, Q}(b)\right) \chi_{Q}\right\|_{L^{s(\cdot)}\left(\mathbb{R}^{n}\right)}}{\left\|\chi_{Q}\right\|_{L^{s(\cdot)}\left(\mathbb{R}^{n}\right)}}<\infty .
$$

(5) For all $s(\cdot) \in \mathscr{B}\left(\mathbb{R}^{n}\right)$ we have (1.2).

Remark 1.3 For the case $\alpha=0$, the result was proved in [27]. Moreover, (1.2) gives a new characterization of nonnegative Lipschitz functions, compared with [27, Theorem 1.5].

For the case of $p(\cdot)$ and $q(\cdot)$ being constants, we have the following result from Theorem 1.1, which is new even for this case.

Corollary 1.1 Let $0<\beta<1,0<\alpha<n, 0<\alpha+\beta<n$ and let $b$ be a locally integrable function. Then the following statements are equivalent:

(1) $b \in \dot{\Lambda}_{\beta}\left(\mathbb{R}^{n}\right)$ and $b \geq 0$.

(2) $\left[b, M_{\alpha}\right]$ is bounded from $L^{p}\left(\mathbb{R}^{n}\right)$ to $L^{q}\left(\mathbb{R}^{n}\right)$ for some $p$ and $q$ such that $1<p<n /(\alpha+\beta)$ and $1 / q=1 / p-(\alpha+\beta) / n$.

(3) $\left[b, M_{\alpha}\right]$ is bounded from $L^{p}\left(\mathbb{R}^{n}\right)$ to $L^{q}\left(\mathbb{R}^{n}\right)$ for all $p$ and $q$ such that $1<p<n /(\alpha+\beta)$ and $1 / q=1 / p-(\alpha+\beta) / n$.

(4) There exists $s \in[1, \infty)$ such that

$$
\sup _{Q} \frac{1}{|Q|^{\beta / n}}\left(\left.\left.\frac{1}{|Q|} \int_{Q}|b(x)-| Q\right|^{-\alpha / n} M_{\alpha, Q}(b)(x)\right|^{s} d x\right)^{1 / s}<\infty .
$$


(5) For all $s \in[1, \infty)$ we have (1.3).

Remark 1.4 The result was proved for $\alpha=0$ in [26, Theorem 1.4]. Corollary 1.1 improves the result of [16, Corollary 4.15] essentially and answers a question asked in [16, Remark 4.17] affirmatively. Moreover, it was proved in [26, Theorem 1.4], see also Lemma 2.2 below, that $b \in \dot{\Lambda}_{\beta}\left(\mathbb{R}^{n}\right)$ and $b \geq 0$ if and only if

$$
\sup _{Q} \frac{1}{|Q|^{\beta / n}}\left(\frac{1}{|Q|} \int_{Q}\left|b(x)-M_{Q}(b)(x)\right|^{s} d x\right)^{1 / s}<\infty .
$$

Compared with (1.4), (1.3) gives a new characterization for nonnegative Lipschitz functions.

Theorem 1.2 Let $0<\alpha<n$ and $b$ be a locally integrable function. Then the following statements are equivalent:

(1) $b \in B M O\left(\mathbb{R}^{n}\right)$ and $b^{-} \in L^{\infty}\left(\mathbb{R}^{n}\right)$.

(2) $\left[b, M_{\alpha}\right]$ is bounded from $L^{p(\cdot)}\left(\mathbb{R}^{n}\right)$ to $L^{q(\cdot)}\left(\mathbb{R}^{n}\right)$ for some $(p(\cdot), q(\cdot)) \in \mathscr{B}^{\alpha}\left(\mathbb{R}^{n}\right)$.

(3) $\left[b, M_{\alpha}\right]$ is bounded from $L^{p(\cdot)}\left(\mathbb{R}^{n}\right)$ to $L^{q(\cdot)}\left(\mathbb{R}^{n}\right)$ for all $(p(\cdot), q(\cdot)) \in \mathscr{B}^{\alpha}\left(\mathbb{R}^{n}\right)$.

(4) There exists $s(\cdot) \in \mathscr{B}\left(\mathbb{R}^{n}\right)$ such that

$$
\sup _{Q} \frac{\left\|\left(b-|Q|^{-\alpha / n} M_{\alpha, Q}(b)\right) \chi_{Q}\right\|_{L^{s \cdot(\cdot)}\left(\mathbb{R}^{n}\right)}}{\left\|\chi_{Q}\right\|_{L^{s(\cdot)}\left(\mathbb{R}^{n}\right)}}<\infty .
$$

(5) For all $s(\cdot) \in \mathscr{B}\left(\mathbb{R}^{n}\right)$ we have (1.5).

Remark 1.5 The equivalence of (1), (2) and (3) was proved in [29]. Statements (4) and (5) give new necessary and sufficient conditions for the statements (1), (2) and (3). Especially, (1.5) gives a new characterization for $b \in B M O\left(\mathbb{R}^{n}\right)$ and $b^{-} \in L^{\infty}\left(\mathbb{R}^{n}\right)$, which also answers a question asked in [29, Remark 4.1]. For the case $\alpha=0$, the result was obtained in [30].

For the case of $p(\cdot)$ and $q(\cdot)$ being constants, we have the following result by Theorem 1.2.

Corollary 1.2 Let $0<\alpha<n$ and $b$ be a locally integrable function. Then the following statements are equivalent:

(1) $b \in B M O\left(\mathbb{R}^{n}\right)$ and $b^{-} \in L^{\infty}\left(\mathbb{R}^{n}\right)$.

(2) $\left[b, M_{\alpha}\right]$ is bounded from $L^{p}\left(\mathbb{R}^{n}\right)$ to $L^{q}\left(\mathbb{R}^{n}\right)$ for some $p$ and $q$ such that $1<p<n / \alpha$ and $1 / q=1 / p-\alpha / n$.

(3) $\left[b, M_{\alpha}\right]$ is bounded from $L^{p}\left(\mathbb{R}^{n}\right)$ to $L^{q}\left(\mathbb{R}^{n}\right)$ for all $p$ and $q$ such that $1<p<n / \alpha$ and $1 / q=1 / p-\alpha / n$

(4) There exists $s \in[1, \infty)$ such that

$$
\sup _{Q}\left(\left.\left.\frac{1}{|Q|} \int_{Q}|b(x)-| Q\right|^{-\alpha / n} M_{\alpha, Q}(b)(x)\right|^{s} d x\right)^{1 / s}<\infty
$$

(5) For all $s \in[1, \infty)$ we have (1.6). 
Remark 1.6 It was shown in [2] and [28] that statements (1), (2) and (3) are equivalent to

$$
\sup _{Q} \frac{1}{|Q|} \int_{Q}\left|b(x)-M_{Q}(b)(x)\right|^{s} d x<\infty,
$$

respectively. Compared with (1.7), (1.6) gives a new characterization.

Next, we give some necessary and sufficient conditions for the boundedness of the maximal commutator $M_{\alpha, b}$ on variable Lebesgue spaces when $b$ belongs to a Lipschitz space.

Theorem 1.3 Let $0<\beta<1,0<\alpha<n, 0<\alpha+\beta<n$ and let $b$ be a locally integrable function. Then the following statements are equivalent:

(1) $b \in \dot{\Lambda}_{\beta}\left(\mathbb{R}^{n}\right)$.

(2) $M_{\alpha, b}$ is bounded from $L^{p(\cdot)}\left(\mathbb{R}^{n}\right)$ to $L^{q(\cdot)}\left(\mathbb{R}^{n}\right)$ for some $(p(\cdot), q(\cdot)) \in \mathscr{B}^{\alpha+\beta}\left(\mathbb{R}^{n}\right)$.

(3) $M_{\alpha, b}$ is bounded from $L^{p(\cdot)}\left(\mathbb{R}^{n}\right)$ to $L^{q(\cdot)}\left(\mathbb{R}^{n}\right)$ for all $(p(\cdot), q(\cdot)) \in \mathscr{B}^{\alpha+\beta}\left(\mathbb{R}^{n}\right)$.

(4) There exists $s(\cdot) \in \mathscr{B}\left(\mathbb{R}^{n}\right)$ such that

$$
\sup _{Q} \frac{1}{|Q|^{\beta / n}} \frac{\left\|\left(b-b_{Q}\right) \chi_{Q}\right\|_{L^{(\cdot)}\left(\mathbb{R}^{n}\right)}}{\left\|\chi_{Q}\right\|_{L^{s(\cdot)}\left(\mathbb{R}^{n}\right)}}<\infty .
$$

(5) For all $s(\cdot) \in \mathscr{B}\left(\mathbb{R}^{n}\right)$ we have (1.8).

Remark 1.7 For the case $\alpha=0$, similar results were given in [26] for Lebesgue spaces with constant exponents, and in [27] for the variable case.

When $p(\cdot)$ and $q(\cdot)$ are constants, we get the following result from Theorem 1.3.

Corollary 1.3 Let $0<\beta<1,0<\alpha<n, 0<\alpha+\beta<n$ and $b$ be a locally integrable function. Then the following statements are equivalent:

(1) $b \in \dot{\Lambda}_{\beta}\left(\mathbb{R}^{n}\right)$.

(2) $M_{\alpha, b}$ is bounded from $L^{p}\left(\mathbb{R}^{n}\right)$ to $L^{q}\left(\mathbb{R}^{n}\right)$ for some $p$ and $q$ such that $1<p<n /(\alpha+\beta)$ and $1 / q=1 / p-(\alpha+\beta) / n$.

(3) $M_{\alpha, b}$ is bounded from $L^{p}\left(\mathbb{R}^{n}\right)$ to $L^{q}\left(\mathbb{R}^{n}\right)$ for all $p$ and $q$ such that $1<p<n /(\alpha+\beta)$ and $1 / q=1 / p-(\alpha+\beta) / n$.

(4) There exists $s \in[1, \infty)$ such that

$$
\sup _{Q} \frac{1}{|Q|^{\beta / n}}\left(\frac{1}{|Q|} \int_{Q}\left|b(x)-b_{Q}\right|^{s} d x\right)^{1 / s}<\infty
$$

(5) For all $s \in[1, \infty)$ we have (1.9).

Remark 1.8 The equivalence of (1), (2) and (3) was proved in [26] (for $\alpha=0$ ) and in [16] (for $0<\alpha<n$ ). The equivalence of (1), (4) and (5) is contained in Lemma 2.1 below.

Finally, for the case of completeness of this paper, we state a result similar to Theorem 1.3 without proof, which can be deduced from [29] and [18].

Theorem 1.4 Let $0<\alpha<n$ and $b$ be a locally integrable function. Then the following statements are equivalent: 
(1) $b \in B M O\left(\mathbb{R}^{n}\right)$.

(2) $M_{\alpha, b}$ is bounded from $L^{p(\cdot)}\left(\mathbb{R}^{n}\right)$ to $L^{q(\cdot)}\left(\mathbb{R}^{n}\right)$ for some $(p(\cdot), q(\cdot)) \in \mathscr{B}^{\alpha}\left(\mathbb{R}^{n}\right)$.

(3) $M_{\alpha, b}$ is bounded from $L^{p(\cdot)}\left(\mathbb{R}^{n}\right)$ to $L^{q(\cdot)}\left(\mathbb{R}^{n}\right)$ for all $(p(\cdot), q(\cdot)) \in \mathscr{B}^{\alpha}\left(\mathbb{R}^{n}\right)$.

(4) There exists $s(\cdot) \in \mathscr{B}\left(\mathbb{R}^{n}\right)$ such that

$$
\sup _{Q} \frac{\left\|\left(b-b_{Q}\right) \chi_{Q}\right\|_{L^{s(\cdot)}\left(\mathbb{R}^{n}\right)}}{\left\|\chi_{Q}\right\|_{L^{s(\cdot)}\left(\mathbb{R}^{n}\right)}}<\infty .
$$

(5) For all $s(\cdot) \in \mathscr{B}\left(\mathbb{R}^{n}\right)$ we have (1.10).

Remark 1.9 We note that Theorem 1.4 follows from [29] and [18] directly. Indeed, the equivalence of (1), (2) and (3) was proved in [29] Theorems 3.1 and 3.2 for $\alpha=0$ and $0<$ $\alpha<n$, respectively, and the equivalence of (1), (4) and (5) was obtained in [18, Lemma 3].

If $p(\cdot)$ and $q(\cdot)$ are constants, we have a result similar to Corollary 1.3. We omit the details.

The remainder of this paper is organized as follows. In the next section, we give some lemmas that will be used later. In Sect. 3, we prove Theorems 1.1, 1.2 and 1.3.

\section{Preliminaries and lemmas}

It is known that Lipschitz space $\dot{\Lambda}_{\beta}\left(\mathbb{R}^{n}\right)$ coincides with some Morrey-Companato space (see, e.g., [20]) and can be characterized by mean oscillation as in the following lemma, which is due to DeVore and Sharpley [9] and Janson, Taibleson and Weiss [20] (see also Paluszyński [24]).

Lemma 2.1 Let $0<\beta<1$ and $1 \leq q<\infty$. Define

$$
\dot{\Lambda}_{\beta, q}\left(\mathbb{R}^{n}\right):=\left\{f \in L_{\mathrm{loc}}^{1}\left(\mathbb{R}^{n}\right):\|f\|_{\dot{\Lambda}_{\beta, q}}=\sup _{Q} \frac{1}{|Q|^{\beta / n}}\left(\frac{1}{|Q|} \int_{Q}\left|f(x)-f_{Q}\right|^{q} d x\right)^{1 / q}<\infty\right\} .
$$

Then, for all $0<\beta<1$ and $1 \leq q<\infty, \dot{\Lambda}_{\beta}\left(\mathbb{R}^{n}\right)=\dot{\Lambda}_{\beta, q}\left(\mathbb{R}^{n}\right)$ with equivalent norms.

From the proof of Theorem 1.4 in [26], we can obtain the following characterization of nonnegative Lipschitz functions.

Lemma 2.2 Let $0<\beta<1$ and $b$ be a locally integrable function. Then the following statements are equivalent:

(1) If $b \in \dot{\Lambda}_{\beta}\left(\mathbb{R}^{n}\right)$ and $b \geq 0$.

(2) For all $1 \leq s<\infty$,

$$
\sup _{Q} \frac{1}{|Q|^{\beta / n}}\left(\frac{1}{|Q|} \int_{Q}\left|b(x)-M_{Q}(b)(x)\right|^{s} d x\right)^{1 / s}<\infty .
$$

(3) (2.1) holds for some $1 \leq s<\infty$.

Proof Since the implication (2) $\Rightarrow$ (3) follows readily, and the implication (3) $\Rightarrow$ (1) was proved in [26, Theorem 1.4], we only need to prove (1) $\Rightarrow(2)$. 
If $b \in \dot{\Lambda}_{\beta}\left(\mathbb{R}^{n}\right)$ and $b \geq 0$, then it follows from [26, Theorem 1.4] that (2.1) holds for all $s$ with $n /(n-\beta)<s<\infty$. Applying Hölder's inequality, we see that (2.1) also holds for $1 \leq s \leq n /(n-\beta)$. So, the implication (1) $\Rightarrow(2)$ is proven.

Lemma 2.3 ([2]) Let b be a locally integrable function. Then the following statements are equivalent:

(1) $b \in B M O\left(\mathbb{R}^{n}\right)$ and $b^{-} \in L^{\infty}\left(\mathbb{R}^{n}\right)$.

(2) There exists $s \in[1, \infty)$ such that

$$
\sup _{Q} \frac{1}{|Q|} \int_{Q}\left|b(x)-M_{Q}(b)(x)\right|^{s} d x<\infty
$$

(3) For all $s \in[1, \infty)$ we have (2.2).

The following strong-type estimate for the fractional maximal function is well known, see [11] or [14] for details.

Lemma 2.4 Let $0<\gamma<n, 1<p<n / \gamma$ and $1 / q=1 / p-\gamma / n$. Then there exists a positive constant $C(n, \gamma, p)$ such that

$$
\left\|M_{\gamma}(f)\right\|_{L^{q}\left(\mathbb{R}^{n}\right)} \leq C(n, \gamma, p)\|f\|_{L^{p}\left(\mathbb{R}^{n}\right)} .
$$

As for the boundedness of the fractional maximal function on variable Lebesgue spaces, the following result was given in [6]; see Corollary 2.12 and Remark 2.13 in [6] for details.

Lemma 2.5 Let $0<\gamma<n, p(\cdot) \in \mathscr{P}\left(\mathbb{R}^{n}\right)$ with $p_{+}<n / \gamma$ and $1 / q(\cdot)=1 / p(\cdot)-\gamma / n$. If $q(\cdot)(n-$ $\gamma) / n \in \mathscr{B}\left(\mathbb{R}^{n}\right)$, then $M_{\gamma}$ is bounded from $L^{p(\cdot)}\left(\mathbb{R}^{n}\right)$ to $L^{q(\cdot)}\left(\mathbb{R}^{n}\right)$.

By Lemma 2.4, if $0<\gamma<n, 1<p<n / \gamma$ and $f \in L^{p}\left(\mathbb{R}^{n}\right)$, then $M_{\gamma}(f)(x)<\infty$ almost everywhere. A similar result is also valid in variable Lebesgue spaces.

Lemma 2.6 Let $0<\gamma<n, p(\cdot) \in \mathscr{P}\left(\mathbb{R}^{n}\right)$ and $1<p_{-} \leq p_{+}<n / \gamma$. If $f \in L^{p(\cdot)}\left(\mathbb{R}^{n}\right)$, then $M_{\gamma}(f)(x)<\infty$ for almost every $x \in \mathbb{R}^{n}$.

Proof Following the same procedure of the proof of [5, Proposition 3.15], we can achieve the desired result. Indeed, for any $f \in L^{p(\cdot)}\left(\mathbb{R}^{n}\right)$, by Theorem 2.51 in [5] we can write $f=$ $f_{1}+f_{2}$, where $f_{1} \in L^{p_{+}}\left(\mathbb{R}^{n}\right)$ and $f_{2} \in L^{p_{-}}\left(\mathbb{R}^{n}\right)$. Then $M_{\gamma}(f)(x) \leq M_{\gamma}\left(f_{1}\right)(x)+M_{\gamma}\left(f_{2}\right)(x)$. Noting that $1<p_{-} \leq p_{+}<n / \gamma$ and $0<\gamma<n$, by Lemma 2.4 we see that $M_{\gamma}\left(f_{1}\right)(x)$ and $M_{\gamma}\left(f_{2}\right)(x)$ are finite almost everywhere. Then $M_{\gamma}(f)(x)<\infty$ for almost every $x \in \mathbb{R}^{n}$.

We also need some basic properties of variable Lebesgue spaces. Denote by $p^{\prime}(\cdot)$ the conjugate index of $p(\cdot)$. Obviously, if $p(\cdot) \in \mathscr{P}\left(\mathbb{R}^{n}\right)$ then $p(\cdot) \in \mathscr{P}\left(\mathbb{R}^{n}\right)$. The following lemma is known as the generalized Hölder's inequality in variable Lebesgue spaces; see [5] and [10] for details. 


\section{Lemma 2.7}

(i) Let $p(\cdot) \in \mathscr{P}\left(\mathbb{R}^{n}\right)$. Then there exists a positive constant $C$ such that for all $f \in L^{p(\cdot)}\left(\mathbb{R}^{n}\right)$ and $g \in L^{p^{\prime}(\cdot)}\left(\mathbb{R}^{n}\right)$

$$
\int_{\mathbb{R}^{n}}|f(x) g(x)| d x \leq C\|f\|_{L^{p \cdot(\cdot)}\left(\mathbb{R}^{n}\right)}\|g\|_{L^{p^{\prime} \cdot(\cdot)\left(\mathbb{R}^{n}\right)}} .
$$

(ii) Let $p(\cdot), p_{1}(\cdot), p_{2}(\cdot) \in \mathscr{P}\left(\mathbb{R}^{n}\right)$ and $1 / p(\cdot)=1 / p_{1}(\cdot)+1 / p_{2}(\cdot)$. Then there exists a positive constant $C$ such that for all $f \in L^{p_{1}(\cdot)}\left(\mathbb{R}^{n}\right)$ and $g \in L^{p_{2}(\cdot)}\left(\mathbb{R}^{n}\right)$,

$$
\|f g\|_{L^{p(\cdot)}\left(\mathbb{R}^{n}\right)} \leq C\|f\|_{L^{p_{1}(\cdot)}\left(\mathbb{R}^{n}\right)}\|g\|_{L^{p_{2}(\cdot)}\left(\mathbb{R}^{n}\right)} \cdot
$$

Lemma 2.8 ([7]) Given $p(\cdot) \in \mathscr{P}\left(\mathbb{R}^{n}\right)$, for all $r>0$ we have

$$
\left\||f|^{r}\right\|_{L^{p(\cdot)}\left(\mathbb{R}^{n}\right)}=\|f\|_{L^{r p(\cdot)}\left(\mathbb{R}^{n}\right)^{\prime}}^{r}
$$

Lemma 2.9 ([17]) Let $p(\cdot) \in \mathscr{B}\left(\mathbb{R}^{n}\right)$, then there exists a constant $C>0$ such that

$$
\frac{1}{|Q|}\left\|\chi_{Q}\right\|_{L^{p(\cdot)}\left(\mathbb{R}^{n}\right)}\left\|\chi_{Q}\right\|_{L^{p^{\prime} \cdot(\cdot)}\left(\mathbb{R}^{n}\right)} \leq C
$$

for all cubes $Q$ in $\mathbb{R}^{n}$.

Lemma 2.10 ([27]) Let $0<\gamma<n, p(\cdot) \in \mathscr{P}\left(\mathbb{R}^{n}\right)$ with $p_{+}<n / \gamma$ and $1 / q(\cdot)=1 / p(\cdot)-\gamma / n$. If $q(\cdot)(n-\gamma) / n \in \mathscr{B}\left(\mathbb{R}^{n}\right)$, then there exists a constant $C>0$ such that

$$
\left\|\chi_{Q}\right\|_{L^{p(\cdot)}\left(\mathbb{R}^{n}\right)} \leq C|Q|^{\gamma / n}\left\|\chi_{Q}\right\|_{L^{q(\cdot)}\left(\mathbb{R}^{n}\right)}
$$

for all cubes $Q$ in $\mathbb{R}^{n}$.

Now, we give the following pointwise estimate for $\left[b, M_{\alpha}\right]$ when $b \in \dot{\Lambda}_{\beta}\left(\mathbb{R}^{n}\right)$.

Lemma 2.11 Let $0 \leq \alpha<n, 0<\beta<1,0<\alpha+\beta<$ nand let $f$ be a locally integrablefunction. If $b \in \dot{\Lambda}_{\beta}\left(\mathbb{R}^{n}\right)$ and $b \geq 0$, then, for any $x \in \mathbb{R}^{n}$ such that $M_{\alpha}(f)(x)<\infty$, we have

$$
\left|\left[b, M_{\alpha}\right] f(x)\right| \leq\|b\|_{\dot{\Lambda}_{\beta}} M_{\alpha+\beta}(f)(x) .
$$

Proof For any fixed $x \in \mathbb{R}^{n}$ such that $M_{\alpha}(f)(x)<\infty$, if $b \geq 0$ and $b \in \dot{\Lambda}_{\beta}\left(\mathbb{R}^{n}\right)$ then

$$
\begin{aligned}
\left|\left[b, M_{\alpha}\right](f)(x)\right| & =\left|b(x) M_{\alpha}(f)(x)-M_{\alpha}(b f)(x)\right| \\
& =\left|\sup _{Q \ni x} \frac{1}{|Q|^{1-\alpha / n}} \int_{Q} b(x)\right| f(y)\left|d y-\sup _{Q \ni x} \frac{1}{|Q|^{1-\alpha / n}} \int_{Q} b(y)\right| f(y)|d y| \\
& \leq \sup _{Q \ni x} \frac{1}{|Q|^{1-\alpha / n}} \int_{Q}|b(x)-b(y)||f(y)| d y \\
& \leq\|b\|_{\dot{A}_{\beta}\left(\mathbb{R}^{n}\right)} \sup _{Q \ni x} \frac{1}{|Q|^{1-(\alpha+\beta) / n}} \int_{Q}|f(y)| d y \\
& \leq\|b\|_{\dot{A}_{\beta}} M_{\alpha+\beta}(f)(x) .
\end{aligned}
$$


Finally, we also need the following result.

Lemma $2.12([2,28])$ Let $0 \leq \gamma<n, Q$ be a cube in $\mathbb{R}^{n}$, and $f$ be a locally integrable function. Then for all $x \in Q$,

$$
M_{\gamma}\left(f \chi_{Q}\right)(x)=M_{\gamma, Q}(f)(x)
$$

and

$$
M_{\gamma}\left(\chi_{Q}\right)(x)=M_{\gamma, Q}\left(\chi_{Q}\right)(x)=|Q|^{\gamma / n} .
$$

\section{Proofs of Theorems 1.1, 1.2 and 1.3}

To prove Theorem 1.1, we first prove the following lemma.

Lemma 3.1 Let $0<\beta<1$ and $0<\gamma<n$. If $b$ is a locally integrable function and satisfies

$$
\sup _{Q} \frac{1}{|Q|^{\beta / n}} \frac{\left\|\left(b-|Q|^{-\gamma / n} M_{\gamma, Q}(b)\right) \chi_{Q}\right\|_{L^{s(\cdot)}\left(\mathbb{R}^{n}\right)}}{\left\|\chi_{Q}\right\|_{L^{s(\cdot)}\left(\mathbb{R}^{n}\right)}} \leq C
$$

for some $s(\cdot) \in \mathscr{B}\left(\mathbb{R}^{n}\right)$, then $b \in \dot{\Lambda}_{\beta}\left(\mathbb{R}^{n}\right)$.

Proof Some ideas are taken from [2, 28] and [29]. Reasoning as the proof of (4.4) in [29], see also the proof of Lemma 2.4 in [28], we have, for any cube $Q$,

$$
\frac{1}{|Q|^{1+\beta / n}} \int_{Q}\left|b(x)-b_{Q}\right| d x \leq\left.\frac{2}{|Q|^{1+\beta / n}} \int_{Q}|b(x)-| Q\right|^{-\gamma / n} M_{\gamma, Q}(b)(x) \mid d x .
$$

Indeed, for any cube $Q$, let $E=\left\{x \in Q: b(x) \leq b_{Q}\right\}$ and $F=\left\{x \in Q: b(x)>b_{Q}\right\}$. It is easy to check that the following equality is true (see [2] page 3331):

$$
\int_{E}\left|b(x)-b_{Q}\right| d x=\int_{F}\left|b(x)-b_{Q}\right| d x .
$$

Noticing the obvious estimate

$$
\left|b_{Q}\right| \leq|Q|^{-\gamma / n} M_{\gamma, Q}(b)(x) \quad \text { for any } x \in Q
$$

and $b(x) \leq b_{Q}$ for any $x \in E$, we have

$$
b(x) \leq b_{Q} \leq\left|b_{Q}\right| \leq|Q|^{-\gamma / n} M_{\gamma, Q}(b)(x) \quad \text { for any } x \in E .
$$

Then, for any $x \in E$,

$$
\left|b(x)-b_{Q}\right| \leq\left.|b(x)-| Q\right|^{-\gamma / n} M_{\gamma, Q}(b)(x) \mid .
$$

Therefore,

$$
\begin{aligned}
\frac{1}{|Q|^{1+\beta / n}} \int_{Q}\left|b(x)-b_{Q}\right| d x & =\frac{1}{|Q|^{1+\beta / n}} \int_{E \cup F}\left|b(x)-b_{Q}\right| d x \\
& =\frac{2}{|Q|^{1+\beta / n}} \int_{E}\left|b(x)-b_{Q}\right| d x
\end{aligned}
$$




$$
\begin{aligned}
& \leq\left.\frac{2}{|Q|^{1+\beta / n}} \int_{E}|b(x)-| Q\right|^{-\gamma / n} M_{\gamma, Q}(b)(x) \mid d x \\
& \leq\left.\frac{2}{|Q|^{1+\beta / n}} \int_{Q}|b(x)-| Q\right|^{-\gamma / n} M_{\gamma, Q}(b)(x) \mid d x .
\end{aligned}
$$

By Lemma 2.7(i), (3.1) and Lemma 2.9, we get

$$
\begin{aligned}
& \frac{1}{|Q|^{1+\beta / n}} \int_{Q}\left|b(x)-b_{Q}\right| d x \\
& \quad \leq \frac{C}{|Q|^{1+\beta / n}}\left\|\left(b-|Q|^{-\gamma / n} M_{\gamma, Q}(b)\right) \chi_{Q}\right\|_{L^{s(\cdot)\left(\mathbb{R}^{n}\right)}}\left\|\chi_{Q}\right\|_{L^{s^{\prime}(\cdot)\left(\mathbb{R}^{n}\right)}} \\
& \quad \leq \frac{C}{|Q|}\left\|\chi_{Q}\right\|_{L^{s(\cdot)\left(\mathbb{R}^{n}\right)}}\left\|\chi_{Q}\right\|_{L^{s^{\prime} \cdot(\cdot)\left(\mathbb{R}^{n}\right)}} \\
& \quad \leq C .
\end{aligned}
$$

So, the proof is completed by applying Lemma 2.1 .

Proof of Theorem 1.1 Since the implications $(3) \Rightarrow(2)$ and $(5) \Rightarrow(4)$ follows readily, we only need to prove (1) $\Rightarrow(3),(2) \Rightarrow(4),(4) \Rightarrow(1)$, and (3) $\Rightarrow(5)$.

(1) $\Rightarrow(3)$. Let $b \in \dot{\Lambda}_{\beta}\left(\mathbb{R}^{n}\right)$ and $b \geq 0$. We need to prove that $\left[b, M_{\alpha}\right]$ is bounded from $L^{p(\cdot)}\left(\mathbb{R}^{n}\right)$ to $L^{q(\cdot)}\left(\mathbb{R}^{n}\right)$ for all $(p(\cdot), q(\cdot)) \in \mathscr{B}^{\alpha+\beta}\left(\mathbb{R}^{n}\right)$. For such $p(\cdot)$ and any $f \in L^{p(\cdot)}\left(\mathbb{R}^{n}\right)$, it follows from Lemma 2.6 that $M_{\alpha}(f)(x)<\infty$ for almost every $x \in \mathbb{R}^{n}$. By Lemma 2.11 , we have

$$
\left|\left[b, M_{\alpha}\right] f(x)\right| \leq\|b\|_{\dot{\Lambda}_{\beta}} M_{\alpha+\beta}(f)(x)
$$

Then, statement (3) follows from Lemma 2.5.

$(2) \Rightarrow(4)$. Let $(p(\cdot), q(\cdot)) \in \mathscr{B}^{\alpha+\beta}\left(\mathbb{R}^{n}\right)$ be such that $\left[b, M_{\alpha}\right]$ is bounded from $L^{p(\cdot)}\left(\mathbb{R}^{n}\right)$ to $L^{q(\cdot)}\left(\mathbb{R}^{n}\right)$. We will verify $(1.2)$ for $s(\cdot)=q(\cdot)$. For any fixed cube $Q$ and any $x \in Q$, it follows from Lemma 2.12 that

$$
M_{\alpha}\left(\chi_{Q}\right)(x)=M_{\alpha, Q}\left(\chi_{Q}\right)(x)=|Q|^{\alpha / n} \quad \text { and } \quad M_{\alpha}\left(b \chi_{Q}\right)(x)=M_{\alpha, Q}(b)(x) .
$$

Then, for any $x \in Q$,

$$
\begin{aligned}
b(x) & -|Q|^{-\alpha / n} M_{\alpha, Q}(b)(x) \\
= & |Q|^{-\alpha / n}\left(b(x)|Q|^{\alpha / n}-M_{\alpha, Q}(b)(x)\right) \\
& =|Q|^{-\alpha / n}\left(b(x) M_{\alpha}\left(\chi_{Q}\right)(x)-M_{\alpha}\left(b \chi_{Q}\right)(x)\right) \\
& =|Q|^{-\alpha / n}\left[b, M_{\alpha}\right]\left(\chi_{Q}\right)(x) .
\end{aligned}
$$

Thus

$$
\left(b(x)-|Q|^{-\alpha / n} M_{\alpha, Q}(b)(x)\right) \chi_{Q}(x)=|Q|^{-\alpha / n}\left[b, M_{\alpha}\right]\left(\chi_{Q}\right)(x) \chi_{Q}(x) .
$$


Noting that $\left[b, M_{\alpha}\right]$ is bounded from $L^{p(\cdot)}\left(\mathbb{R}^{n}\right)$ to $L^{q(\cdot)}\left(\mathbb{R}^{n}\right)$ with $1 / q(\cdot)=1 / p(\cdot)-(\alpha+\beta) / n$ and applying Lemma 2.10 , we have

$$
\begin{aligned}
& \left\|\left(b-|Q|^{-\alpha / n} M_{\alpha, Q}(b)\right) \chi_{Q}\right\|_{L^{q(\cdot)}\left(\mathbb{R}^{n}\right)} \\
& \leq|Q|^{-\alpha / n}\left\|\left[b, M_{\alpha}\right]\left(\chi_{Q}\right)\right\|_{L^{q(\cdot)}\left(\mathbb{R}^{n}\right)} \\
& \leq C|Q|^{-\alpha / n}\left\|\left[b, M_{\alpha}\right]\right\|_{L^{p(\cdot)} \rightarrow L^{q(\cdot)}}\left\|\chi_{Q}\right\|_{L^{p^{(\cdot)}\left(\mathbb{R}^{n}\right)}} \\
& \leq C|Q|^{\beta / n}\left\|\left[b, M_{\alpha}\right]\right\|_{L^{p(\cdot)} \rightarrow L^{q(\cdot)}}\left\|\chi_{Q}\right\|_{L^{q(\cdot)}\left(\mathbb{R}^{n)}\right.},
\end{aligned}
$$

which gives (1.2) for $s(\cdot)=q(\cdot)$ since $Q$ is arbitrary and $C$ is independent of $Q$.

$(4) \Rightarrow(1)$. By Lemma 2.2, it suffices to prove

$$
\sup _{Q} \frac{1}{|Q|^{1+\beta / n}} \int_{Q}\left|b(x)-M_{Q}(b)(x)\right| d x<\infty .
$$

For any fixed cube $Q$,

$$
\begin{aligned}
& \frac{1}{|Q|^{1+\beta / n}} \int_{Q}\left|b(x)-M_{Q}(b)(x)\right| d x \\
& \leq\left.\frac{1}{|Q|^{1+\beta / n}} \int_{Q}|b(x)-| Q\right|^{-\alpha / n} M_{\alpha, Q}(b)(x) \mid d x \\
& \quad+\left.\frac{1}{|Q|^{1+\beta / n}} \int_{Q}|| Q\right|^{-\alpha / n} M_{\alpha, Q}(b)(x)-M_{Q}(b)(x) \mid d x \\
& :=I_{1}+I_{2} .
\end{aligned}
$$

For $I_{1}$, by statement (4) and applying Lemma 2.7(i) and Lemma 2.9, we have

$$
\begin{aligned}
I_{1} & \leq \frac{C}{|Q|^{1+\beta / n}}\left\|\left(b-|Q|^{-\alpha / n} M_{\alpha, Q}(b)\right) \chi_{Q}\right\|_{L^{s \cdot(\cdot)}\left(\mathbb{R}^{n}\right)}\left\|\chi_{Q}\right\|_{L^{s^{\prime}(\cdot)}\left(\mathbb{R}^{n}\right)} \\
& \leq \frac{C}{|Q|^{\beta / n}} \frac{\left\|\left(b-|Q|^{-\alpha / n} M_{\alpha, Q}(b)\right) \chi_{Q}\right\|_{L^{s \cdot(\cdot)\left(\mathbb{R}^{n}\right)}}}{\left\|\chi_{Q}\right\|_{\left.L^{s \cdot \cdot(}\right)\left(\mathbb{R}^{n}\right)}} \\
& \leq C
\end{aligned}
$$

where the constant $C$ is independent of $Q$.

Next, we consider $I_{2}$. Similar to the proof of Theorem 1.1 in [31], we can get $I_{2} \leq C$. Now, we give the proof of this. For all $x \in Q$, it follows from Lemma 2.12 that

$$
M\left(\chi_{Q}\right)(x)=\chi_{Q}(x)=1 \text { and } M\left(b \chi_{Q}\right)(x)=M_{Q}(b)(x),
$$

and

$$
M_{\alpha}\left(\chi_{Q}\right)(x)=|Q|^{\alpha / n} \quad \text { and } \quad M_{\alpha}\left(b \chi_{Q}\right)(x)=M_{\alpha, Q}(b)(x) .
$$

Then, for any $x \in Q$,

$$
\begin{aligned}
& \left.|| Q\right|^{-\alpha / n} M_{\alpha, Q}(b)(x)-M_{Q}(b)(x) \mid \\
& \quad \leq\left.|Q|^{-\alpha / n}\left|M_{\alpha, Q}(b)(x)-\right| Q\right|^{\alpha / n}|b(x)||+||b(x)|-M_{Q}(b)(x) \mid
\end{aligned}
$$




$$
\begin{aligned}
\leq & |Q|^{-\alpha / n}\left|M_{\alpha}\left(b \chi_{Q}\right)(x)-\right| b(x)\left|M_{\alpha}\left(\chi_{Q}\right)(x)\right| \\
& +|| b(x)\left|M\left(\chi_{Q}\right)(x)-M\left(b \chi_{Q}\right)(x)\right| \\
\leq & |Q|^{-\alpha / n}\left|\left[|b|, M_{\alpha}\right]\left(\chi_{Q}\right)(x)\right|+\left|[|b|, M]\left(\chi_{Q}\right)(x)\right| .
\end{aligned}
$$

Since $s(\cdot) \in \mathscr{B}\left(\mathbb{R}^{n}\right)$, statement (4) along with Lemma 3.1 gives $b \in \dot{\Lambda}_{\beta}\left(\mathbb{R}^{n}\right)$, which implies $|b| \in \dot{\Lambda}_{\beta}\left(\mathbb{R}^{n}\right)$. Thus, we can apply Lemma 2.11 to $\left[|b|, M_{\alpha}\right]$ and $[|b|, M]$ since $|b| \in \dot{\Lambda}_{\beta}\left(\mathbb{R}^{n}\right)$ and $|b| \geq 0$. By Lemmas 2.11 and 2.12, we have, for any $x \in Q$,

$$
\left|\left[|b|, M_{\alpha}\right]\left(\chi_{Q}\right)(x)\right| \leq\|b\|_{\dot{\Lambda}_{\beta}} M_{\alpha+\beta}\left(\chi_{Q}\right)(x) \leq C\|b\|_{\dot{\Lambda}_{\beta}}|Q|^{(\alpha+\beta) / n}
$$

and

$$
\left|[|b|, M]\left(\chi_{Q}\right)(x)\right| \leq\|b\|_{\dot{\Lambda}_{\beta}} M_{\beta}\left(\chi_{Q}\right)(x) \leq C\|b\|_{\dot{\Lambda}_{\beta}}|Q|^{\beta / n} .
$$

By (3.4), we have

$$
\begin{aligned}
I_{2}= & \left.\frac{1}{|Q|^{1+\beta / n}} \int_{Q}|| Q\right|^{-\alpha / n} M_{\alpha, Q}(b)(x)-M_{Q}(b)(x) \mid d x \\
\leq & \frac{C}{|Q|^{1+(\alpha+\beta) / n}} \int_{Q}\left|\left[|b|, M_{\alpha}\right]\left(\chi_{Q}\right)(x)\right| d x \\
& +\frac{C}{|Q|^{1+\beta / n}} \int_{Q}\left|[|b|, M]\left(\chi_{Q}\right)(x)\right| d x \\
\leq & C\|b\|_{\dot{A}_{\beta}} .
\end{aligned}
$$

Putting the above estimates for $I_{1}$ and $I_{2}$ into (3.3), we obtain (3.2).

$(3) \Rightarrow(5)$. Assume statement (3) is true. Reasoning as in the proof of (2) $\Rightarrow(4)$, we have

$$
\sup _{Q} \frac{1}{|Q|^{\beta / n}} \frac{\left\|\left(b-|Q|^{-\alpha / n} M_{\alpha, Q}(b)\right) \chi_{Q}\right\|_{L^{q(\cdot)}\left(\mathbb{R}^{n}\right)}}{\left\|\chi_{Q}\right\|_{L^{q(\cdot)}\left(\mathbb{R}^{n}\right)}}<\infty
$$

for any $q(\cdot)$ for which there exists a $p(\cdot)$ such that $(p(\cdot), q(\cdot)) \in \mathscr{B}^{\alpha+\beta}\left(\mathbb{R}^{n}\right)$.

For any $s(\cdot) \in \mathscr{B}\left(\mathbb{R}^{n}\right)$, choosing an $r>n /(n-\beta)$, we have $r s(\cdot)(n-\beta) / n \in \mathscr{B}\left(\mathbb{R}^{n}\right)$ and $r s(\cdot) \in \mathscr{B}\left(\mathbb{R}^{n}\right)$ by Remark 1.1. Set $q(\cdot)=r s(\cdot)$ and define $p(\cdot)$ by $1 / p(\cdot)=1 / q(\cdot)+(\alpha+\beta) / n$. It is easy to check that $(p(\cdot), q(\cdot)) \in \mathscr{B}^{\alpha+\beta}\left(\mathbb{R}^{n}\right)$.

Noting that

$$
\frac{1}{s(\cdot)}=\frac{1}{r s(\cdot)}+\frac{1}{r^{\prime} s(\cdot)}=\frac{1}{q(\cdot)}+\frac{1}{r^{\prime} s(\cdot)}
$$

it follows from Lemma 2.7(ii), (3.5) and Lemma 2.8 that

$$
\begin{aligned}
& \frac{1}{|Q|^{\beta / n}} \frac{\left\|\left(b-|Q|^{-\alpha / n} M_{\alpha, Q}(b)\right) \chi_{Q}\right\|_{L^{s(\cdot)}\left(\mathbb{R}^{n}\right)}}{\left\|\chi_{Q}\right\|_{L^{s \cdot(\cdot)}\left(\mathbb{R}^{n}\right)}} \\
& \quad \leq \frac{1}{|Q|^{\beta / n}} \frac{\left\|\left(b-|Q|^{-\alpha / n} M_{\alpha, Q}(b)\right) \chi_{Q}\right\|_{L^{q \cdot(\cdot)}\left(\mathbb{R}^{n}\right)}\left\|\chi_{Q}\right\|_{L^{r^{\prime}(\cdot)}\left(\mathbb{R}^{n}\right)}}{\left\|\chi_{Q}\right\|_{L^{s(\cdot)}\left(\mathbb{R}^{n}\right)}}
\end{aligned}
$$




$$
\begin{aligned}
& \leq \frac{C\left\|\chi_{Q}\right\|_{L^{q(\cdot)}\left(\mathbb{R}^{n}\right)}\left\|\chi_{Q}\right\|_{L^{r^{\prime}(\cdot)\left(\mathbb{R}^{n}\right)}}}{\left\|\chi_{Q}\right\|_{L^{s \cdot(\cdot)}\left(\mathbb{R}^{n}\right)}} \\
& =\frac{C\left\|\chi_{Q}\right\|_{L^{s(\cdot)}\left(\mathbb{R}^{n}\right)}^{1 / r}\left\|\chi_{Q}\right\|_{L^{s(\cdot)}\left(\mathbb{R}^{n}\right)}^{1 / r^{\prime}}}{\left\|\chi_{Q}\right\|_{L^{s(\cdot)}\left(\mathbb{R}^{n}\right)}}=C,
\end{aligned}
$$

which is what we want.

The proof of Theorem 1.1 is finished.

Remark 3.1 The proof of (3) $\Rightarrow(5)$ is also valid for $\beta=0$.

To prove Theorem 1.2, we recall the following results obtained in [29].

\section{Lemma 3.2}

(1) Let $p(\cdot) \in \mathscr{B}\left(\mathbb{R}^{n}\right)$.If $0 \leq b \in B M O\left(\mathbb{R}^{n}\right)$, then $[b, M]$ is bounded from $L^{p(\cdot)}\left(\mathbb{R}^{n}\right)$ to itself.

(2) Let $0<\gamma<n, p(\cdot) \in \mathscr{P}\left(\mathbb{R}^{n}\right)$ with $p_{+}<n / \gamma, 1 / q(\cdot)=1 / p(\cdot)-\gamma / n$ and $q(\cdot) /(n-\gamma) \in \mathscr{B}\left(\mathbb{R}^{n}\right)$. If $0 \leq b \in B M O\left(\mathbb{R}^{n}\right)$, then $\left[b, M_{\alpha}\right]$ is bounded from $L^{p(\cdot)}\left(\mathbb{R}^{n}\right)$ to $L^{q(\cdot)}\left(\mathbb{R}^{n}\right)$.

The following result can be deduced from the proof of Lemma 4.1 in [29].

Lemma 3.3 Let $0<\gamma<n$. If $b$ is a locally integrable function and satisfies

$$
\sup _{Q} \frac{\left\|\left(b-|Q|^{-\gamma / n} M_{\gamma, Q}(b)\right) \chi_{Q}\right\|_{L^{s(\cdot)}\left(\mathbb{R}^{n}\right)}}{\left\|\chi_{Q}\right\|_{L^{s \cdot(\cdot)}\left(\mathbb{R}^{n}\right)}}<\infty
$$

for some $s(\cdot) \in \mathscr{B}\left(\mathbb{R}^{n}\right)$, then $b \in B M O\left(\mathbb{R}^{n}\right)$.

Proof of Theorem 1.2 Since the equivalence of (1), (2) and (3) was given in [29, Theorem 1.1], the implication (2) $\Rightarrow$ (4) follows from [29, Lemma 4.1] and (3) $\Rightarrow$ (5) follows from Remark 3.1, we only need to prove the implication $(4) \Rightarrow(1)$.

For any fixed cube $Q$, it follows from (3.3) and (3.4) that

$$
\begin{aligned}
\frac{1}{|Q|} \int_{Q}\left|b(x)-M_{Q}(b)(x)\right| d x \leq & \left.\frac{1}{|Q|} \int_{Q}|b(x)-| Q\right|^{-\alpha / n} M_{\alpha, Q}(b)(x) \mid d x \\
& +\frac{1}{|Q|^{1+\alpha / n}} \int_{Q}\left|\left[|b|, M_{\alpha}\right]\left(\chi_{Q}\right)(x)\right| d x \\
& +\frac{1}{|Q|} \int_{Q}\left|[|b|, M]\left(\chi_{Q}\right)(x)\right| d x \\
:= & J_{1}+J_{2}+J_{3} .
\end{aligned}
$$

For $J_{1}$, by Lemma 2.7(i), Lemma 2.9 and statement (4), we have

$$
\begin{aligned}
J_{1} & \leq \frac{C}{|Q|}\left\|\left(b-|Q|^{-\alpha / n} M_{\alpha, Q}(b)\right) \chi_{Q}\right\|_{L^{s \cdot(\cdot)}\left(\mathbb{R}^{n}\right)}\left\|\chi_{Q}\right\|_{L^{s^{\prime}(\cdot)}\left(\mathbb{R}^{n}\right)} \\
& \leq \frac{C\left\|\left(b-|Q|^{-\alpha / n} M_{\alpha, Q}(b)\right) \chi_{Q}\right\|_{L^{s \cdot(\cdot)}\left(\mathbb{R}^{n}\right)}}{\left\|\chi_{Q}\right\|_{L^{s \cdot \cdot}\left(\mathbb{R}^{n}\right)}} \\
& \leq C,
\end{aligned}
$$

where the constant $C$ is independent of $Q$. 
Set $q(\cdot)=s(\cdot) n /(n-\alpha)$. By Remark 1.1, we have $q(\cdot) \in \mathscr{B}\left(\mathbb{R}^{n}\right)$ since $s(\cdot) \in \mathscr{B}\left(\mathbb{R}^{n}\right)$. For a $p(\cdot)$ such that $1 / q(\cdot)=1 / p(\cdot)-\alpha / n$, we have $p(\cdot) \in \mathscr{P}\left(\mathbb{R}^{n}\right)$ and $p_{+}<n / \alpha$.

Noticing that $s(\cdot) \in \mathscr{B}\left(\mathbb{R}^{n}\right)$, statement (4) along with Lemma 3.3 gives $b \in B M O\left(\mathbb{R}^{n}\right)$, which implies $|b| \in B M O\left(\mathbb{R}^{n}\right)$. Thus, we can apply Lemma 3.2 to $\left[|b|, M_{\alpha}\right]$ and $[|b|, M]$ for the pair of exponents $p(\cdot)$ and $q(\cdot)$ given as above and get

$$
\left\|[|b|, M]\left(\chi_{Q}\right)\right\|_{L^{p(\cdot)}\left(\mathbb{R}^{n}\right)} \leq C\left\|\chi_{Q}\right\|_{L^{p(\cdot)}\left(\mathbb{R}^{n}\right)}
$$

and

$$
\left\|\left[|b|, M_{\alpha}\right]\left(\chi_{Q}\right)\right\|_{L^{q(\cdot)}\left(\mathbb{R}^{n}\right)} \leq C\left\|\chi_{Q}\right\|_{L^{p(\cdot)}\left(\mathbb{R}^{n}\right)} .
$$

Then, it follows from Lemma 2.7(i), Lemma 2.10 and Lemma 2.9 that

$$
\begin{aligned}
J_{2} & =\frac{1}{|Q|^{1+\alpha / n}} \int_{Q}\left|\left[|b|, M_{\alpha}\right]\left(\chi_{Q}\right)(x)\right| d x \\
& \leq \frac{C}{|Q|^{1+\alpha / n}}\left\|\left[|b|, M_{\alpha}\right]\left(\chi_{Q}\right)\right\|_{L^{q(\cdot)\left(\mathbb{R}^{n}\right)}}\left\|\chi_{Q}\right\|_{L^{q^{\prime}(\cdot)\left(\mathbb{R}^{n}\right)}} \\
& \leq \frac{C}{|Q|^{1+\alpha / n}}\left\|\chi_{Q}\right\|_{L^{p(\cdot)}\left(\mathbb{R}^{n}\right)}\left\|\chi_{Q}\right\|_{L^{q^{\prime}(\cdot)\left(\mathbb{R}^{n}\right)}} \\
& \leq \frac{C}{|Q|}\left\|\chi_{Q}\right\|_{L^{q(\cdot)}\left(\mathbb{R}^{n}\right)}\left\|\chi_{Q}\right\|_{L^{q^{\prime} \cdot(\cdot)\left(\mathbb{R}^{n}\right)}} \\
& \leq C .
\end{aligned}
$$

Similarly, by Lemma 2.7(i) and Lemma 2.9, we have

$$
\begin{aligned}
J_{3} & =\frac{1}{|Q|} \int_{Q}\left|[|b|, M]\left(\chi_{Q}\right)(x)\right| d x \\
& \leq \frac{C}{|Q|}\left\|[|b|, M]\left(\chi_{Q}\right)\right\|_{L^{p(\cdot)\left(\mathbb{R}^{n}\right)}}\left\|\chi_{Q}\right\|_{L^{p^{\prime} \cdot(\cdot)\left(\mathbb{R}^{n}\right)}} \\
& \leq \frac{C}{|Q|}\left\|\chi_{Q}\right\|_{L^{p(\cdot)}\left(\mathbb{R}^{n}\right)}\left\|\chi_{Q}\right\|_{L^{p^{\prime} \cdot(\cdot)\left(\mathbb{R}^{n}\right)}} \\
& \leq C .
\end{aligned}
$$

Putting the above estimates for $J_{1}, J_{2}$ and $J_{3}$ into (3.6), we obtain

$$
\frac{1}{|Q|} \int_{Q}\left|b(x)-M_{Q}(b)(x)\right| d x \leq C,
$$

which implies $b \in B M O\left(\mathbb{R}^{n}\right)$ and $b^{-} \in L^{\infty}\left(\mathbb{R}^{n}\right)$ by Lemma 2.3 , since the constant $C$ is independent of $Q$.

The proof of Theorem 1.2 is completed.

Proof of Theorem 1.3 Since the equivalence of (1), (4) and (5) were proved in [27, Corollary 1.1], we only need to prove the implications $(1) \Rightarrow(3)$ and $(2) \Rightarrow(4)$. 
(1) $\Rightarrow$ (3). If $b \in \dot{\Lambda}_{\beta}\left(\mathbb{R}^{n}\right)$, then

$$
\begin{aligned}
M_{\alpha, b}(f)(x) & =\sup _{Q \ni x} \frac{1}{|Q|^{1-\alpha / n}} \int_{Q}|b(x)-b(y)||f(y)| d y \\
& \leq C\|b\|_{\dot{A}_{\beta}} \sup _{Q \ni x} \frac{1}{|Q|^{1-(\alpha+\beta) / n}} \int_{Q}|f(y)| d y \\
& =C\|b\|_{\dot{\Lambda}_{\beta}} M_{\alpha+\beta}(f)(x) .
\end{aligned}
$$

This, together with Lemma 2.5 , shows that $M_{\alpha, b}$ is bounded from $L^{p(\cdot)}\left(\mathbb{R}^{n}\right)$ to $L^{q(\cdot)}\left(\mathbb{R}^{n}\right)$.

(2) $\Rightarrow(4)$. For any fixed cube $Q$, we have for all $x \in Q$,

$$
\begin{aligned}
\left|b(x)-b_{Q}\right| & \leq \frac{1}{|Q|} \int_{Q}|b(x)-b(y)| d y \\
& =\frac{1}{|Q|} \int_{Q}|b(x)-b(y)| \chi_{Q}(y) d y \\
& \leq|Q|^{-\alpha / n} M_{\alpha, b}\left(\chi_{Q}\right)(x) .
\end{aligned}
$$

Then, for all $x \in \mathbb{R}^{n}$,

$$
\left|\left(b(x)-b_{Q}\right) \chi_{Q}(x)\right| \leq|Q|^{-\alpha / n} M_{\alpha, b}\left(\chi_{Q}\right)(x) .
$$

Since $M_{\alpha, b}$ is bounded from $L^{p(\cdot)}\left(\mathbb{R}^{n}\right)$ to $L^{q(\cdot)}\left(\mathbb{R}^{n}\right)$, by Lemma 2.10 , we have

$$
\begin{aligned}
\left\|\left(b-b_{Q}\right) \chi_{Q}\right\|_{L^{q(\cdot)}\left(\mathbb{R}^{n}\right)} & \leq|Q|^{-\alpha / n}\left\|M_{\alpha, b}\left(\chi_{Q}\right)\right\|_{L^{q(\cdot)}\left(\mathbb{R}^{n}\right)} \\
& \leq C\left\|M_{\alpha, b}\right\|_{L^{p(\cdot)} \rightarrow L^{q(\cdot)}}|Q|^{-\alpha / n}\left\|\chi_{Q}\right\|_{L^{p(\cdot)}\left(\mathbb{R}^{n}\right)} \\
& \leq C\left\|M_{\alpha, b}\right\|_{L^{p(\cdot)} \rightarrow L^{q(\cdot)}}|Q|^{\beta / n}\left\|\chi_{Q}\right\|_{L^{q(\cdot)}\left(\mathbb{R}^{n}\right)},
\end{aligned}
$$

which gives (1.8) for $s(\cdot)=q(\cdot)$ since $Q$ is arbitrary and $C$ is independent of $Q$.

The proof of Theorem 1.3 is finished.

\section{Acknowledgements}

The authors are grateful to the referee for careful reading of the paper and valuable suggestions and comments.

\section{Funding}

The first author is supported by the National Natural Science Foundation of China (Grant Nos. 11571160, 11471176) and the Scientific Research Fund of Mudanjiang Normal University (No. MSB201201). The second author is supported by the Key Research Project for Higher Education in Henan Province (No. 19A110017).

\section{Competing interests}

The authors declare that they have no competing interests.

Authors' contributions

All authors read and approved the final manuscript.

\section{Author details}

'Department of Mathematics, Mudanjiang Normal University, Mudanjiang, P.R. China. ${ }^{2}$ School of Mathematics and Information Science, Henan Polytechnic University, Jiaozuo, P.R. China.

\section{Publisher's Note}

Springer Nature remains neutral with regard to jurisdictional claims in published maps and institutional affiliations.

Received: 14 August 2018 Accepted: 6 January 2019 Published online: 15 January 2019 


\section{References}

1. Agcayazi, M., Gogatishvili, A., Koca, K., Mustafayev, R.: A note on maximal commutators and commutators of maximal functions. J. Math. Soc. Jpn. 67(2), 581-593 (2015)

2. Bastero, J., Milman, M., Ruiz, F.J.: Commutators for the maximal and sharp functions. Proc. Am. Math. Soc. 128(11), 3329-3334 (2000)

3. Bonami, A., Iwaniec, T., Jones, P., Zinsmeister, M.: On the product of functions in $B M O$ and $H^{1}$. Ann. Inst. Fourier (Grenoble) 57(5), 1405-1439 (2007)

4. Coifman, R.R., Rochberg, R., Weiss, G.: Factorization theorems for Hardy spaces in several variables. Ann. Math. 103, 611-635 (1976)

5. Cruz-Uribe, D., Fiorenza, A.: Variable Lebesgue Spaces: Foundations and Harmonic Analysis. Springer, Heidelberg (2013)

6. Cruz-Uribe, D., Fiorenza, A., Martell, J.M., Pérez, C.: The boundedness of classical operators on variable $L^{p}$ spaces. Ann Acad. Sci. Fenn., Math. 31, 239-264 (2006)

7. Cruz-Uribe, D., Wang, L.-A.D.: Variable Hardy spaces. Indiana Univ. Math. J. 63(2), 447-493 (2014)

8. Deringoz, F., Guliyev, V.S., Hasanov, S.G.: Commutators of fractional maximal operator on generalized Orlicz-Morrey spaces. Positivity 22, 141-158 (2018)

9. DeVore, R.A., Sharpley, R.C.: Maximal functions measuring smoothness. Mem. Am. Math. Soc. 47(293), viii+115 (1984)

10. Diening, L., Harjulehto, P., Hästö, P., Ružǐčka, M.: Lebesgue and Sobolev Spaces with Variable Exponents. Lecture Notes in Math., vol. 2017. Springer, Heidelberg (2011)

11. Duoandikoetxea, J.: Fourier Analysis. Graduate Studies in Math., vol. 29. Am. Math. Soc., Providence (2001)

12. Fan, Y., Jia, H.Y.: Boundedness of commutators of maximal function on Morrey space. Acta Math. Sin., Chin. Ser. 55(4), 701-706 (2012)

13. Gogatishvili, A., Mustafayev, R., Ağcayazi, M.: Weak-type estimates in Morrey spaces for maximal commutator and commutator of maximal function. Tokyo J. Math. 41(1), 193-218 (2018)

14. Grafakos, L.: Modern Fourier Analysis, 3rd edn. Graduate Texts in Math., vol. 250. Springer, New York (2014)

15. Guliyev, V.S., Deringoz, F.: Some characterizations of Lipschitz spaces via commutators on generalized Orlicz-Morrey spaces. Mediterr. J. Math. 15(4), Article ID 180 (2018). https://doi.org/10.1007/s00009-018-1226-5

16. Guliyev, V.S., Deringoz, F., Hasanov, S.G.: Fractional maximal function and its commutators on Orlicz spaces. Anal. Math. Phys. (2017). https://doi.org/10.1007/s13324-017-0189-1

17. Izuki, M.: Boundedness of sublinear operators on Herz spaces with variable exponent and application to wavelet characterization. Anal. Math. 36, 33-50 (2010)

18. Izuki, M.: Boundedness of commutators on Herz spaces with variable exponent. Rend. Circ. Mat. Palermo 59, 199-213 (2010)

19. Janson, S.: Mean oscillation and commutators of singular integral operators. Ark. Mat. 16, 263-270 (1978)

20. Janson, S. Taibleson, M. Weiss, G.: Elementary characterization of the Morrey-Campanato spaces. In: Harmonic Analysis (Cortona, 1982). Lecture Notes in Math., vol. 992, pp. 101-114. Springer, Berlin (1983)

21. Kokilashvili, V., Meskhi, A., Rafeiro, H., Samko, S.: In: Integral Operators in Non-standard Function Spaces, Vol. 1: Variable Exponent Lebesgue and Amalgam Spaces. Operator Theory: Advances and Applications, vol. 248. Birkhäuser, Basel (2016)

22. Kokilashvili, V., Meskhi, A., Rafeiro, H., Samko, S.: Integral Operators in Non-standard Function Spaces, Vol. 2: Variable Exponent Hölder, Morrey-Campanato and Grand Spaces. Operator Theory: Advances and Applications, vol. 249. Birkhäuser, Basel (2016)

23. Milman, M., Schonbek, T.: Second order estimates in interpolation theory and applications. Proc. Am. Math. Soc. 110(4), 961-969 (1990)

24. Paluszyński, M.: Characterization of the Besov spaces via the commutator operator of Coifman, Rochberg and Weiss. Indiana Univ. Math. J. 44(1), 1-17 (1995)

25. Xie, C.P.: Some estimates of commutators. Real Anal. Exch. 36(2), 405-416 (2010/2011)

26. Zhang, P.: Characterization of Lipschitz spaces via commutators of the Hardy-Littlewood maximal function. C. R. Math. 355(3), 336-344 (2017)

27. Zhang, P.: Characterization of boundedness of some commutators of maximal functions in terms of Lipschitz spaces. Anal. Math. Phys. (2018). https://doi.org/10.1007/s13324-018-0245-5

28. Zhang, P., Wu, J.L.: Commutators of the fractional maximal functions. Acta Math. Sin., Chin. Ser. 52(6), 1235-1238 (2009)

29. Zhang, P., Wu, J.L.: Commutators of the fractional maximal function on variable exponent Lebesgue spaces. Czechoslov. Math. J. 64, 183-197 (2014)

30. Zhang, P., Wu, J.L.: Commutators for the maximal functions on Lebesgue spaces with variable exponent. Math. Inequal. Appl. 17(4), 1375-1386 (2014)

31. Zhang, P., Wu, J.L., Sun, J.: Commutators of some maximal functions with Lipschitz function on Orlicz spaces. Mediterr. J. Math. 15(6), Article ID 216 (2018). https://doi.org/10.1007/s00009-018-1263-0 